\title{
Automated Synthesis of a Human-Competitive Solution to the Challenge Problem of the 2002 International Optical Design Conference by Means of Genetic Programming and a Multi-Dimensional Mutation Operation
}

\author{
Lee W. Jones \\ Genetic Programming Inc. \\ 990 Villa Street \\ Mountain View, California 94041 \\ lee@genetic-programming.com
}

\author{
Sameer H. Al-Sakran \\ Genetic Programming Inc. \\ 990 Villa Street \\ Mountain View, California 94041 \\ sameer@genetic-programming.com
}

\author{
John R. Koza \\ Stanford University \\ Stanford, California 94305 \\ PHONE: 650-960-8180 \\ koza@stanford.edu
}

\begin{abstract}
This paper has two aspects. First, it describes the use of genetic programming to automatically synthesize a solution to the challenge problem posed at an international competition held every four years in the field of optical design. In 2002, the competition at the International Optical Design Conference attracted 42 entries from 39 well-known optical designers, commercial consultants, and patent holders from many of the field's most prominent companies, universities, and research institutions. The 39 human contestants spent an average of 34.1 hours working on their entries. Virtually all entries were considered good solutions to the challenge problem. Genetic programming automatically synthesized a design "from scratch" - that is, without starting from a pre-existing humancreated design and without pre-specifying the number of lenses, the physical layout of the lenses, or the numerical or nonnumerical parameters of the lenses. The run of genetic programming did not employ any knowledge base of design techniques or principles from the field of optical design and did not entail any human intervention during the run. The genetically evolved optical lens system would have ranked in the middle $\left(21^{\text {st }}\right)$ if it had been entered into the 2002 competition and is therefore an instance of a "human-competitive" result produced by genetic programming. Second, this paper presents a mutation operation for numerical constants that is especially appropriate for problems in which the to-be-designed structure contains a large number of non-linearly interrelated numerical values and for problems in which the topology of the solution is to be automatically created.
\end{abstract}

\section{Categories and Subject Descriptors}

G.1.6-Global Optimization; I.2.2-Automatic Programming Program Synthesis; I.2.8-Control Methods and Search; J.2Physics

\section{General Terms \\ Design, algorithms}

Permission to make digital or hard copies of all or part of this work for personal or classroom use is granted without fee provided that copies are not made or distributed for profit or commercial advantage and that copies bear this notice and the full citation on the first page. To copy otherwise, or republish, to post on servers or to redistribute to lists, requires prior specific permission and/or a fee. GECCO'06, July 8-12, 2006, Seattle, Washington, USA. Copyright 2006 ACM 1-59593-186-4/06/0007...\$5.00.

\section{Keywords}

Genetic programming, automated design, optical lens system, human-competitive result, invention machine, mutation operation, International Optical Design Conference

\section{INTRODUCTION}

Every four years, a design competition is held at the International Optical Design Conference (IODC). In his paper reporting on the 2002 competition, Richard C. Juergens of Raytheon Missile Systems described the goal of the challenge problem formulated by the competition's six-member committee of optical design experts [8]:

\footnotetext{
The problem ideally should not be a commercially viable lens (this is to encourage participation in the problem), should be optically challenging, should be one that cannot be solved easily with a global optimizer, and should not favor one lens design program over another by its requirements.
}

In 2002, 42 entries were submitted by 39 human contestants. Most contestants were well-known optical designers, commercial consultants, and patent holders from many of the most prominent companies, universities, and research institutions in the field of optical design. The contestants were given several months to formulate their solutions to the challenge problem. The contestants reported spending an average of 34.1 hours working on their entries. Participants in the competitions each had between 5 and 40 years of experience in the field of optical design [4].

Juergen [8] summarized the 42 entries by saying:

The different design forms submitted show that different designers used different techniques and different optimization methodologies to solve the problem....

This problem showed a wide variation among entrants in design form, the number of lenses used, glass choice, overall length, etc. So rather than being a test of optical design codes, it was more of a test of the designers. This was a problem where designer ingenuity and "thinking outside of the box" was essential to getting a good solution. 
[I]n general, the results were quite good, with most of the spot sizes well below the diffraction limit, and with the RMS [root mean square] focal shift differences being much less than $0.001 \mathrm{~mm}$. [Emphasis added]

In addition to Juergens, the committee included Richard Pfisterer of Photon Engineering, Richard Youngworth of Kodak, Joseph Howard of NASA Goddard Space Flight Center, Yongtian Wang of the Beijing Institute of Technology, and Bany Johnson of Optical E.T.C. David Shafer of Shafer Lens Design came up with the idea for the problem. The committee was advised by Paul Manhart of Raytheon Missile Systems and Robert Shannon of the University of Arizona Optical Sciences Center.

This paper has two aspects. First, it describes the use of genetic programming to automatically synthesize a solution to the challenge problem posed at the 2002 International Optical Design Conference. Our evolutionary approach differed from the approach used by the 39 human contestants in that it was fully automated. Genetic programming automatically synthesized a design "from scratch"- that is, without starting from a pre-existing human-created design and without prespecifying the number of lenses, the physical layout of the lenses, or the numerical or non-numerical parameters of the lenses. The run of genetic programming did not employ any knowledge base of design techniques or principles from the field of optical design and did not entail any human intervention during the run. The human contestants generally started from design that they thought were good and then applied their judgment and standard software optimization techniques (e.g., damped least squares) to improve their designs.

The evolved optical lens system would have ranked in the middle $\left(21^{\text {st }}\right)$ if it had been entered into the 2002 IODC competition. The evolved lens system is therefore an instance of a "human-competitive" result $[12,13]$ produced by genetic programming

Second, this paper presents a mutation operation for numerical constants that is especially appropriate for problems in which the to-be-designed structure contains a large number of nonlinearly interrelated numerical values and in which the topology (that is, the size and shape) of the problem's solution must be automatically created.

Section 2 provides a statement of the problem. Section 3 mentions previous work involving the use of genetic programming to design optical lens systems. Section 4 describes the improved mutation operation for numerical constants. Section 5 discusses the preparatory steps used to apply genetic programming to the automated design of optical lens systems. Section 6 presents the results produced by genetic programming for the challenge problem from the 2002 International Optical Design Conference. Section 7 is the conclusion.

\section{STATEMENT OF THE PROBLEM}

Optical design is more of an art than a science. As Warren J. Smith states in Modern Optical Engineering [16, page 393]:

There is no "direct" method of optical design for original systems; that is, there is no sure procedure that will lead (without foreknowledge) from a set of performance specifications to a suitable design.
A complete design for a classical optical lens system encompasses numerous decisions, including the choice of the system's topology (e.g., the number of lens surfaces, number of lenses, number of lens groups, and their physical placement relative to one another), choices for numerical parameters (e.g., radius of curvature, distance between lens surfaces), and choices for non-numerical parameters (e.g., material). A classical lens system is conventionally specified by a table called a prescription (or, if the system is being analyzed by modern-day optical simulation software, a lens file). Once a classical optical system is specified by means of its prescription or lens file, many of its optical properties can be calculated by tracing the path of light rays of various wavelengths through the system.

The challenge problem at the 2002 International Optical Design Conference called for the design of an all-refractive optical lens system that simulates the chromatic behavior of a diffractive optical element. Diffractive elements have large amounts of axial chromatic aberration - typically reversed in sign of that of normal optical materials and one to two orders of magnitude greater. Specifically, the 2002 problem involved designing an $\mathrm{f} / 8$ lens system with a focal length of $100 \mathrm{~mm}$ at the reference wavelength of $575 \mathrm{~nm}$ that simulates the chromatic nature of a diffractive optical element over wavelengths ranging from 400 to $750 \mathrm{~nm}$. The design was open-ended in that there were no restrictions on the number of lenses or the overall length of the lens system. The following specifications applied:

- The optical lens system was to be all-refractivewith no reflecting or diffractive surfaces allowed.

- Lenses were to be all spherical, and must be made of glasses found in the current or previous Schott glass catalog.

- 15 wavelengths equally spaced across the spectral band were to be used in the evaluation (namely $400,425,450,475,500,525,550,575,600$, $625,650,675,700,725$, and $750 \mathrm{~nm}$ ), with the reference wavelength being $575 \mathrm{~nm}$.

- The differences in paraxial focal shifts at each of the 15 wavelengths from that of the reference wavelength were to be compared to the focal shifts of an ideal diffractive surface. All paraxial focus distances were to be positive from the last glass surface in the system (i.e., no negative image distances).

- Axial and edge glass thicknesses were to be positive.

The IODC 2002 committee specified a particular merit function (what practitioners of genetic and evolutionary computation would call the fitness measure) that was to be optimized and that would be used in ranking entries from the contestants. The IODC fitness measure had two elements. The first element was the RMS (root mean square) of the spot size at the nominal wavelength at a user-specified focal plane (which can be called the imaging performance). The second element was the RMS of the focal shift differences between the lens and those of the diffractive surface (which can be called the diffractive performance). Because the committee believed that it would be considerably easier to achieve a small spot size than to achieve good focal shift differences, the RMS of the spot size was weighted by a factor of 100 relative to the RMS of the focal shift differences. The fitness measure for the 2002 IODC problem was thus 


$$
F=\sqrt{(100 A)^{2}+B^{2}}
$$

In this expression, $A$ is the RMS of the spot size. The value of $A$ is computed with a rectangular grid across the entrance pupil with 100 rays across the diameter (giving 7,850 rays in the onaxis spot diagram).

In the above expression, $B$ is the RMS of the focal shift differences. $S_{\mathrm{i}}$, is the focal shift for the i-th wavelength for the ideal diffractive element that is being approximated in this problem. If $\lambda(i)$ is the wavelength, then

$$
S_{i}=100\left(\frac{575}{\lambda(i)}-1\right) \text {. }
$$

The value of $B$ is calculated from $S_{\mathrm{i}}$, and two measured values of the system, namely $L_{575}$ and $L_{\lambda(\mathrm{i})} L_{575}$ is the measured effective focal length (EFL) of the system at the nominal wavelength of $575 \mathrm{~nm}$. In our approach to the problem, the EFL is scaled so that $L_{575}$ is always $100 \mathrm{~mm}$. $L_{\lambda(\mathrm{i})}$ is the measured EFL of the system at wavelength $\lambda(i)$.

$$
B=\left[\frac{\sum_{i=1}^{15}\left[\left(L_{\lambda(i)}-L_{575}\right)-S_{i}\right]^{2}}{15}\right]^{\frac{1}{2}}
$$

\section{PREVIOUS USE OF GENETIC PROGRAMMING FOR OPTICAL DESIGN}

In 2002, Beaulieu, Gagné, and Parizeau [2] successfully used genetic programming to "re-engineer" the design of a preexisting monochromatic lens system with four lenses using functions that incrementally adjusted (additively or multiplicatively) the distance between lens surfaces, radius of curvature of lens surfaces, and stop location values.

In 2005, Al-Sakran, Koza and Jones [1], Jones, Al-Sakran, and Koza [6, 7] and Koza, Al-Sakran, and Jones [9, 10, 11] used genetic programming to automatically re-invent seven previously patented optical lens systems "from scratch."

\section{MULTI-DIMENSIONAL MUTATION OPERATION}

The challenge of the field of optical design is similar to the challenge involved in designing electrical circuits, antennas, controllers, networks of chemical reactions, and other complex structures in that a satisfactory design usually entails the synthesis of both (1) a topological arrangement of components and (2) a determination of the numerical and non-numerical parameters for each component. Practical design of optical systems differs, however, from the design of these other types of structures in two important respects.

First, in many lens systems, each ray that is used to analyze the system's performance passes through all the system's surfaces and is very substantially influenced by the thickness of each lens, the material of each lens, the curvature of each lens surface, and the spacing between each lens surface. That is, the degree of coupling between the components of an optical system is unusually high.

Second, very small changes in the value of the numerical parameters of the components in an optical system are often very important. That is, sensitivity to component values is unusually high in optical systems. In contrast, most electrical circuits can successfully perform their desired functions even though their individual components are, in practice, manufactured using processes in which component values routinely vary by $5 \%$ or more.

Moreover, it was clear in advance that the demanding specifications of the challenge problem at the 2002 International Optical Design Conference would necessitate a structure having a large number of components - each with numerous numerical and non-numerical parameters. This expectation was confirmed to us when our initial debugging runs yielded structures with well over a hundred parameter values. This expectation was further confirmed by the fact that, among the 42 human entries to the competition, the winning entry had 59 lenses; the largest number of lenses was 90; and the average was 21 lenses. Each surface is characterized by two numerical parameters and one non-numerical parameter. The largest number of lens groups was 31 . The average number of glass types was 8 .

The foregoing considerations suggested that a difficult optical design problem would probably challenge the ability of genetic programming to discover the appropriate value for the numerous numerical and non-numerical values associated with the system's numerous components to a far greater degree than was the case for design problems from other fields that were previously solved by means of genetic programming. This observation suggested that it might be desirable to enhance the ability of genetic programming to cope with design problems involving discovery of numerous highly coupled, highly sensitive parameter values.

The mutation operation typically used in runs of genetic programming for parameter values is a point mutation operation - that is, it adjusts one numerical or non-numerical parameter at a time. In generation 0 , each perturbable value is set, individually and separately, to a random value in a chosen range appropriate for the problem. In the case of problems of optical design, the perturbable value is numerical if a radius of curvature or a distance (thickness) is involved, but nonnumerical if a material is involved (e.g., a type of glass from a specified glass catalog, air, vacuum, oil, plastic). After generation 0 , a perturbable value may be perturbed. For numerical values, the to-be-perturbed value is considered to be the mean of a Gaussian distribution. A relatively small preset parameter establishes the standard deviation of the Gaussian distribution. The to-be-perturbed numerical value is then perturbed by an amount determined by the Gaussian distribution. When a material (e.g., a type of glass) must be mutated in an optical lens system, a two-dimensional mutation operation is used. The current material is considered to be a point on a plane defined by the material's index of refraction, $n$, and its Abbe number, $V$. The to-be-perturbed material is then perturbed by picking a randomly chosen distance determined by a Gaussian distribution. A point in the plane representing an allowable material (e.g., a material in the specified glass catalog) is found whose distance from the point representing the original 
material is closest (measured by Euclidean distance) to the randomly chosen distance.

It is true that successive mutations may sometimes be applied to the same individual. It is also true that, in some versions of genetic programming, two points in a given individual may be mutated (with probability $p^{2}$ ) and $k$ points may be mutated (with probability $p^{\mathrm{k}}$ ). Nonetheless, because $p$ is always relatively small, $p^{2}$ and $p^{\mathrm{k}}$ are exceedingly small. In short, as a practical matter, mutation in genetic programming (and, indeed, in genetic algorithms) performs a low-dimensionality local search - not a high-dimensionality search.

The authors believe that a higher-dimensional mutation operation is appropriate for difficult problems requiring the discovery of numerous highly coupled parameter values. Accordingly, for the work described in this paper, we used a new multi-dimensional mutation operation. This operation is similar to conventional mutation in that it acts on a single individual selected from the population based on its fitness and in that a point is picked at random from the individual in the same manner as for crossover. Figure 1 shows an illustrative individual in which the picked point is the PROG2 function at the top of the sub-tree contained within the dotted lines. In the new multi-dimensional mutation operation, none of the functions in the sub-tree rooted at the picked point are affected by the operation. That is, the new operation does not replace the pre-existing subtree with a newly grown subtree at the picked point. The new operation does not affect the functions in the subtree. Instead, all the parameter values (numerical and nonnumerical) contained in the sub-tree are separately and individually mutated (using the same Gaussian perturbation used for point mutation). During the course of a run of genetic programming, this new multi-dimensional mutation operation is applied to subtrees containing differing numbers of parameters. That is, the dimensionality of the new multi-dimensional mutation operation varies.

In addition to the new multi-dimensional mutation operation described above, this work also employed a domain-specific operation that splits nested groups of lenses. The group-splitting operation begins by randomly picking a surface within a nested group (if any) of lenses. The operation duplicates the picked surface and inserts a fixed amount air (in fact, the minimum allowed amount of air) between the two surfaces. This operation is motivated by a technique described by Smith [15, page 25] often used by optical designers.

There are several classic design modification techniques which can be reliably used to improve an existing lens design [including] split[ing] a cemented doublet.

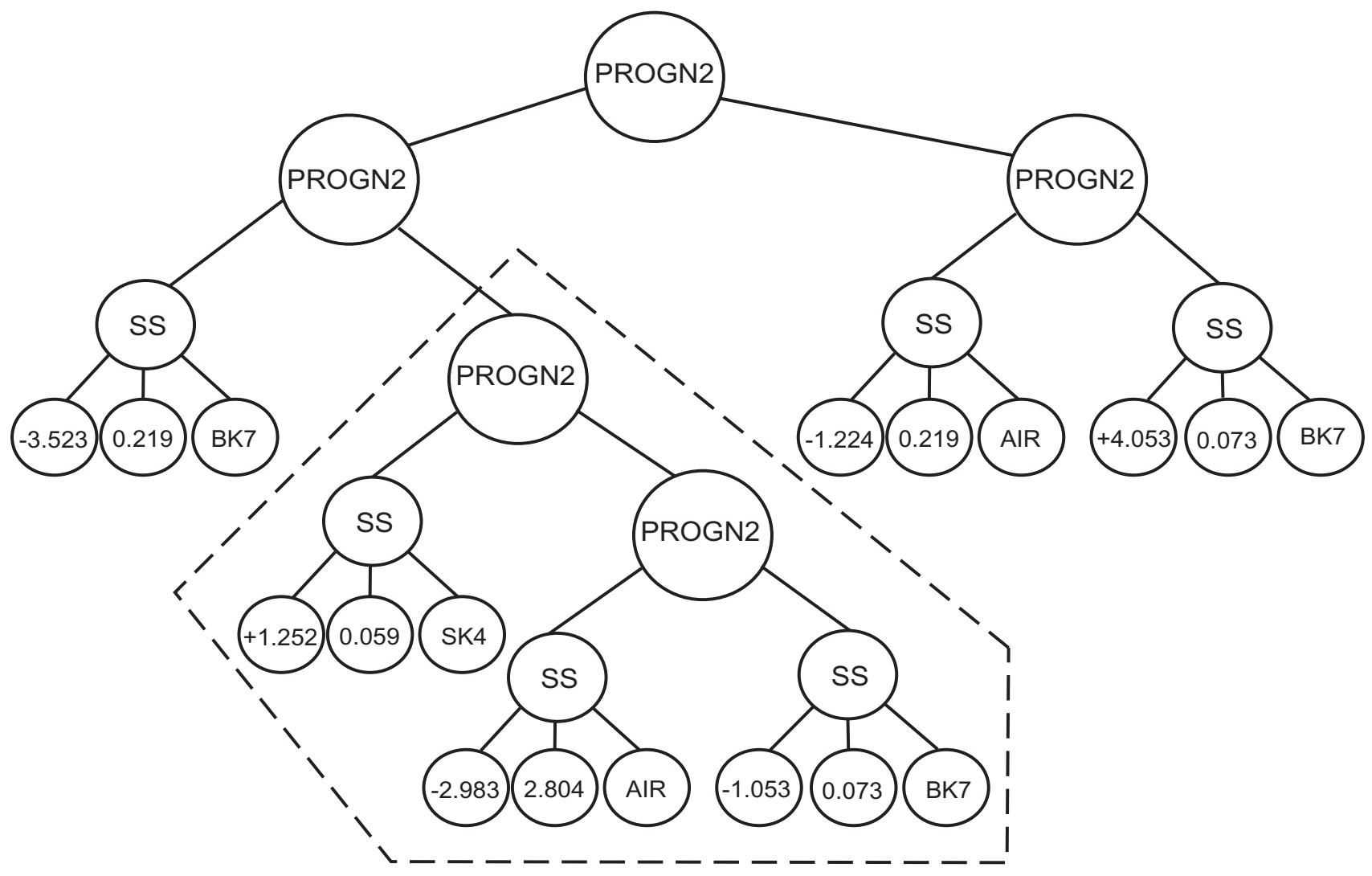

Figure 1 Multi-dimensional mutation operation 


\section{PREPARATORY STEPS}

We now describe the representation scheme (including a developmental process, function set, and terminal set), the fitness measure, and the two domain-specific mutation operations that we used.

\subsection{Representation Scheme}

The format for optical prescriptions (lens files) that is widely used in the field of optical design suggests the use of a developmental process $[17,5]$ for representing individuals in the population. The developmental representation that we used employs a turtle similar to the turtle used in Lindenmayer systems [14], the LOGO programming language, and previous work using genetic programming to synthesize geometric patterns [9], analog electrical circuits [12, 13], and antennas [3]. For work in this paper, we followed our previously published developmental representation, function set, terminal set, constrained syntactic structure, and test fixture $[1,6,7,10]$.

There are two functions (SS and PROGN2) in the function set for this problem. The three-argument SS ("spherical surface") function causes the turtle to do three things at its starting point (and each subsequent point to which the turtle moves). First, it inserts a spherical surface with a specified radius of curvature at the turtle's present location. Second, the SS function moves the turtle to the right by a specified distance along the system's main axis. Third, the SS function fills the space to the right of the just-added surface with a specified type of material. The two-argument PROGN2 function is a connective function.

A constrained syntactic structure specifies how the functions and terminals may be combined in a program tree. The constrained syntactic structure enforces the use of one terminal set (containing perturbable numerical values) for each valuesetting subtree that establishes the numerical value for thickness and radius of curvature; another terminal set for establishing the type of material (containing symbolic values changeable by the glass mutation operation); and the function set for all other parts of the program tree (with PROGN2 as the top-most function in each program tree).

The object surface, image surface and entry pupil together constitute a test fixture that is directly analogous to the test fixtures used in connection with previously published work on the automatic synthesis of electrical circuits by means of genetic programming $[12,13]$.

\subsection{Fitness Measure}

We developed our own lens analysis simulator based on KOJAC, a public-domain educational software package for optical ray tracing originally authored by Olivier Scherler and currently maintained by Olivier Ripoll, to evaluate the performance of candidate lens systems. We wrote code to use the ray traces produced by KOJAC to compute relevant optical characteristics and additionally wrote code for the image analysis. We embedded all of this code into our genetic programming code. For post-run validation of final results, we used a commercially available software package (OSLO from Lambda Research) running on a single workstation.
The fitness of an individual in the population was evaluated in four phases. The first three phases tested whether an individual was degenerate, optically implausible, or was not in the desired result space.

In the first phase, lens systems containing only air surfaces are culled.

In the second phase, the lens system is scaled such that the EFL is exactly $100 \mathrm{~mm}$ when calculated at the nominal wavelength of $575 \mathrm{~nm}$. If the resulting scaled system has a negative EFL or the system becomes extremely large or small, the individual is assigned a large penalty value of fitness (and effectively culled). Otherwise, the focal shift RMS error ( $B$ in section 2$)$ is calculated. The individual is assigned a temporary value of fitness equal to $B$. If $B>0.5$ (i.e., there is insufficient diffractive behavior), the individual is not evaluated further.

In the third phase, the system was checked to be sure that it forms an image to the right of the lenses (i.e., is focal). If the system is focal, no additional value is added to the individual's temporary value of fitness. If the individual was afocal (i.e., the rays exiting the system are divergent), then a value equal to the slope of a divergent reference ray is added to the fitness. Phase 3 is necessary because the calculation of an RMS spot size is meaningless for a system without an image plane. If an individual is compliant with the requirements of the first three phases, we proceed to the fourth phase.

In the fourth phase, 7,850 rays evenly spaced over the entry pupil of the system were cast for the spot diagram and the resultant value of $A$ (the RMS spot size) is calculated. The individual was then assigned a final value of fitness using the formula (described in section 2 above) established by the committee that formulated the challenge problem for the 2002 International Optical Design Conference.

\subsection{Control Parameters}

The population size was approximately $161,600 \quad(320$ individuals per node and 505 nodes on a Beowulf-style cluster computer). The multi-dimensional mutation for numerical and non-numerical values (described in section 4) was performed with a probability of $50 \%$ and the conventional crossover operation for genetic programming [13] was performed with a probability of $35 \%$. The lens-spitting operation [6] and the $n$-let splitting mutation operation (described in section 4) were performed with a probability of $6 \%$ and $5 \%$, respectively. The conventional sub-tree mutation and reproduction operations for genetic programming [13] were performed with probability $1 \%$ and $3 \%$, respectively.

\section{RESULTS}

The best-of-run individual appeared in generation 1019. This individual had a fitness of 0.09042 (i.e., a focal shift RMS value of 0.09152 and a spot size RMS value of 0.00007593 ).

This individual had 88 surfaces (not counting stacked air surfaces) and 76 surfaces (after combining like glass types). The length of the system was $1712.14 \mathrm{~mm}$.

The value of 0.00007593 is two orders of magnitude below the diffraction limit of the evolved system (0.005918). 
(W)

(A)

(B)

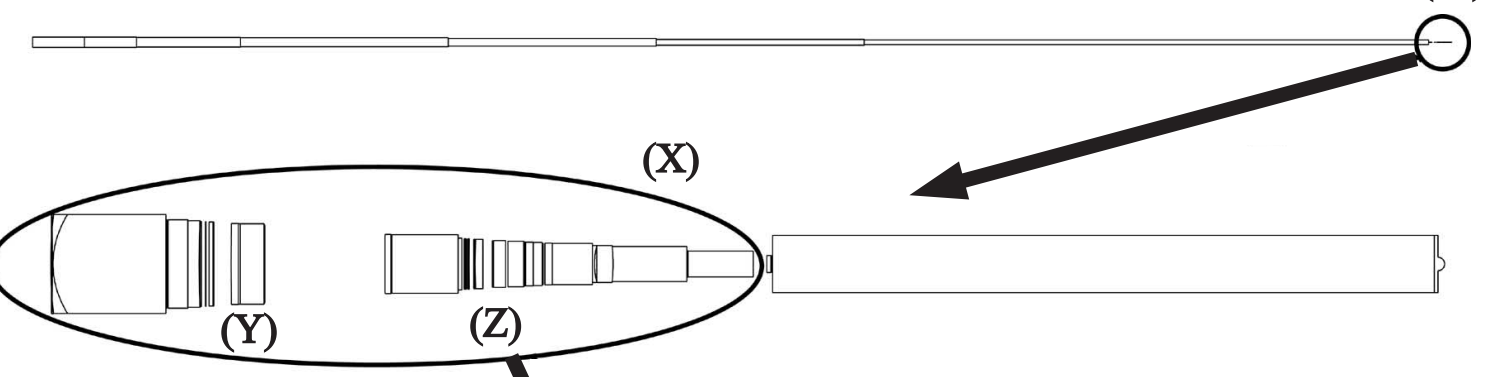

(C)
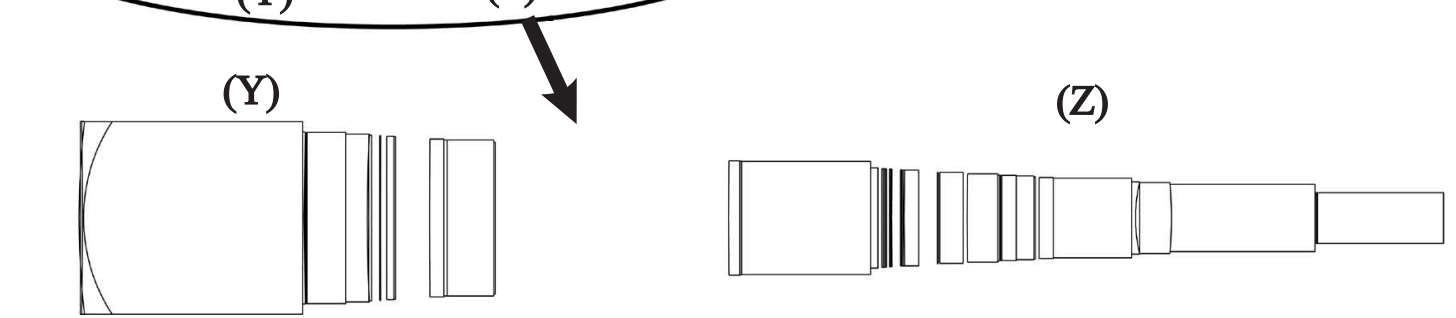

Figure 2 Best-of-run individual from generation 1019

(a)

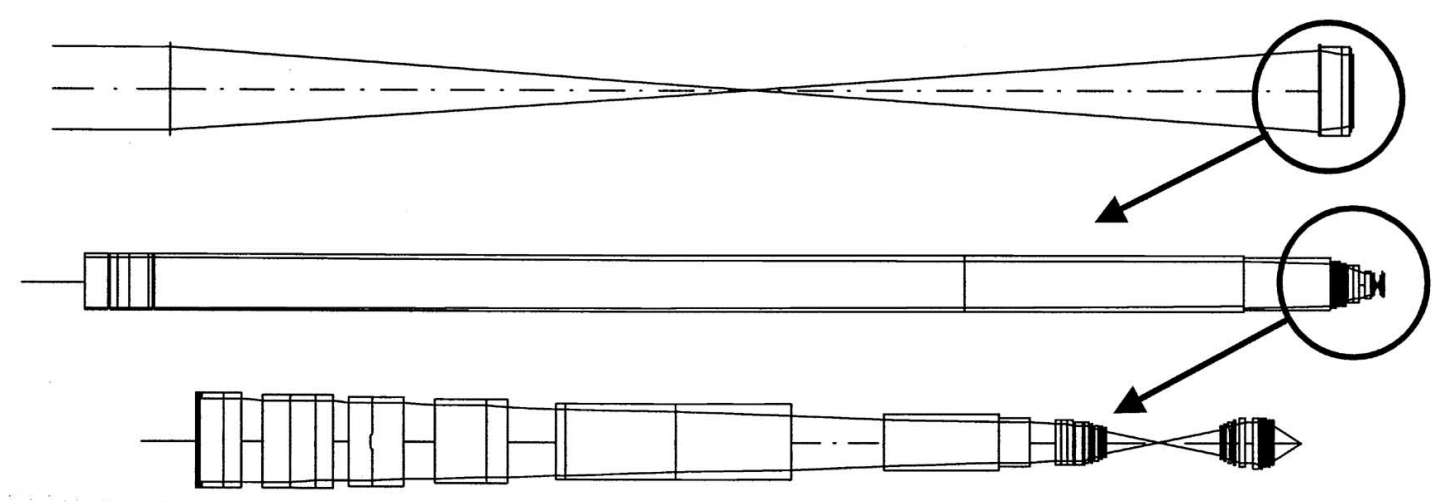

Figure 3 Best result (by Lerner) on the challenge problem at the 2002 International Optical Design Conference

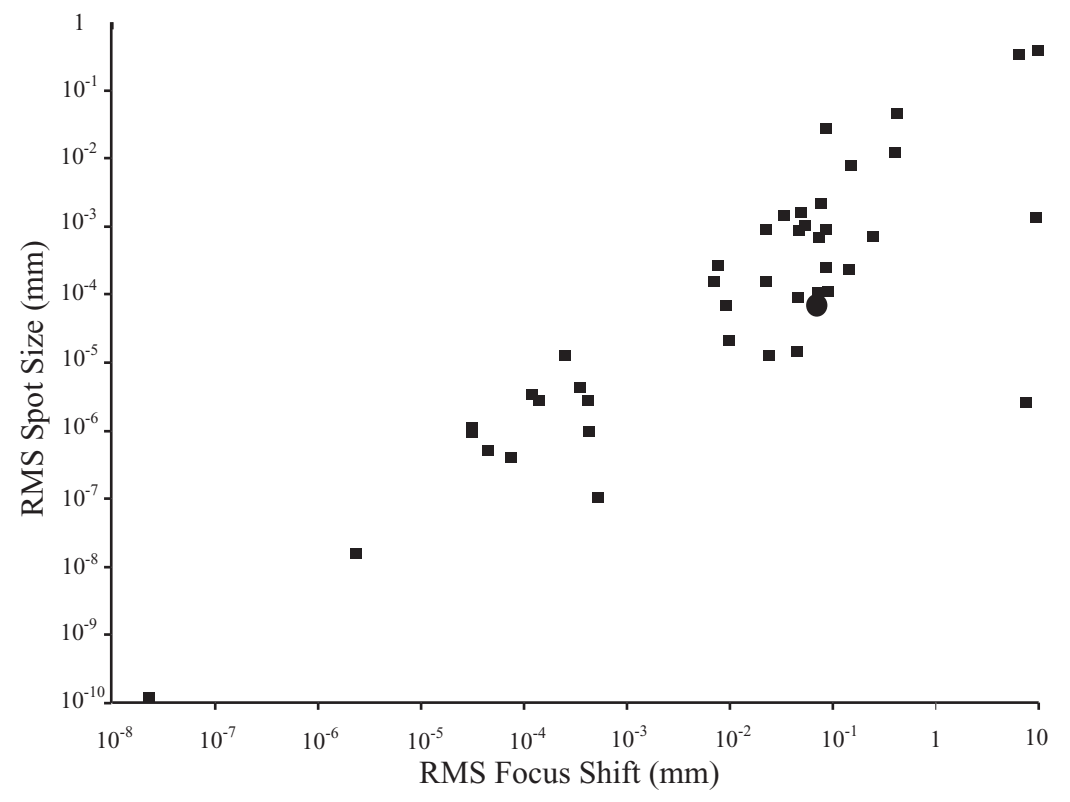

Figure 4 Plot of 42 human-created entries (small squares) and our evolved system (large circle) 
Table 1 compares, for each of the 15 wavelengths, the ideal value of the effective focal length (EFL) with the EFL for the best-of-run individual. Column 2 of the table is the value of the focal length of the ideal diffractive element that is being approximated at each wavelength in this problem. Column 3 is the corresponding value for the best-of-run individual.

Table 1: Comparison of ideal value of effective focal length and evolved individual

\begin{tabular}{|l|l|l|}
\hline Wavelength (nm) & $\begin{array}{l}\text { Ideal } \\
\text { EFL } \\
\text { value }\end{array}$ & $\begin{array}{l}\text { Evolved } \\
\text { EFL } \\
\text { value }\end{array}$ \\
\hline 400 & 143.7514 & 143.696 \\
\hline 425 & 135.2955 & 135.421 \\
\hline 450 & 127.7791 & 127.807 \\
\hline 475 & 121.0538 & 120.977 \\
\hline 500 & 115.0012 & 114.879 \\
\hline 525 & 109.5249 & 109.415 \\
\hline 550 & 104.5465 & 104.485 \\
\hline 575 & 100.001 & 100.001 \\
\hline 600 & 95.83429 & 95.891 \\
\hline 625 & 92.11176 & 92.096 \\
\hline 650 & 88.46242 & 88.569 \\
\hline 675 & 85.18604 & 85.272 \\
\hline 700 & 82.14368 & 82.176 \\
\hline 725 & 79.31114 & 79.256 \\
\hline 750 & 76.66743 & 76.492 \\
\hline
\end{tabular}

Figure 5 shows the same information as table 1. The two sets of values are so similar that the two curves are virtually indistinguishable.

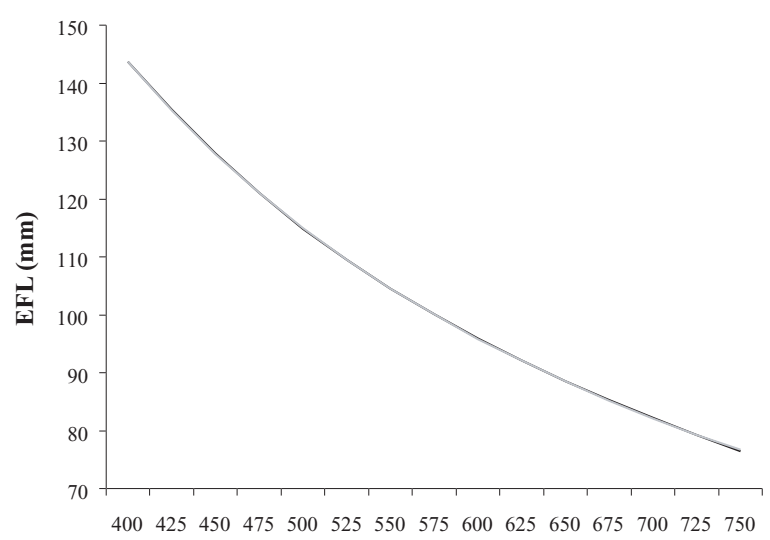

Wavelength (nm)

Figure 5 Comparison of ideal value of effective focal length and evolved individual

Figure 2 presents the optical lens system that emerged as the best-of-run individual from generation 1019. Because lens surfaces in our evolved system (and other entries in the IODC competition) contain several densely concentrated sets of surfaces, the system is presented visually in three parts. Part A of the figure shows the entire optical lens system. The entire evolved system has 76 effective surfaces and a length of 1,712 $\mathrm{mm}$ (about $51 / 2$ feet). Then, the first seven surfaces of part A are deleted and the remaining 69 surfaces (occupying only 28.22 $\mathrm{mm}$ and labeled $\mathrm{W}$ ) are expanded and shown as part B of the figure. Finally, part B's final seven surfaces are deleted and the 62 remaining surfaces (occupying only $14.38 \mathrm{~mm}$ and labeled $Y$ and $\mathrm{Z}$ ) are shown in part $\mathrm{C}$ of the figure. The portion of part $\mathrm{B}$ labeled $\mathrm{Y}$ is shown at the left of part $\mathrm{C}$. The portion of part $\mathrm{B}$ labeled $\mathrm{Z}$ is shown at the right of part $\mathrm{C}$.

The best result on the challenge problem at the 2002 International Optical Design Conference was submitted by Scott Lerner of Lawrence Livermore Labs. Lerner's lens system (figure 3 ) was about $4 / 5$ of a mile $(1,078,241 \mathrm{~mm})$.

Figure 4 is a plot of the 42 entries in the challenge problem at the 2002 International Optical Design Conference in terms of the competition's two judging criteria-namely the RMS focal shift (horizontal axis) and the RMS spot size (vertical axis). Smaller values are better. Lerner's result is in the lower-left corner. Our evolved optical lens system is represented by the large circle in the figure. As can be seen, our evolved optical lens system would have ranked in the middle $\left(21^{\text {st }}\right)$ if it had been entered into the competition in 2002.

Two of the eight criteria $[12,13]$ for saying that an automatically created result is "human-competitive" are

(G) The result solves a problem of indisputable difficulty in its field.

(H) The result holds its own or wins a regulated competition involving human contestants (in the form of either live human players or human-written computer programs).

Our result on the challenge problem at the 2002 International Optical Design Conference satisfies criteria G and H. Therefore, we claim that the genetically evolved result in this paper is an instance of "human-competitive" result produced by genetic programming.

\section{CONCLUSION}

This paper described how genetic programming was used to automatically synthesize a solution to the challenge problem at the 2002 International Optical Design Conference. Genetic programming automatically synthesized a design "from scratch"- that is, without starting from a pre-existing humancreated design and without pre-specifying the number of lenses, the physical layout of the lenses, or the numerical or nonnumerical parameters of the lenses. The automatic synthesis did not employ any knowledge base of principles or techniques from the field of optical design and did not entail any human intervention during the run. The genetically evolved optical lens system would have ranked in the middle $\left(21^{\text {st }}\right)$ if it had been entered into the 2002 competition and is therefore an instance of a "human-competitive" result produced by genetic programming. This paper also described a new highdimensionality mutation operation for numerical constants that is especially appropriate for problems in which the to-bedesigned structure contains a large number of non-linearly interrelated numerical values and in which the topology (that is, the size and shape) of the problem's solution must be automatically created. 


\section{REFERENCES}

1 Al-Sakran, Sameer H., Koza, John R., and Jones, Lee W. 2005. Automated re-invention of a previously patented optical lens system using genetic programming. In Keijzer, Maarten, Tettamanzi, Andrea, Collet, Pierre, van Hemert, Jano, Tomassini, Marco (editor). Genetic Programming: 8th European Conference, EuroGP 2005, Lausanne, Switzerland, March 30-April 1, 2005, Proceedings. Lecture Notes in Computer Science 3447. Heidelberg: SpringerVerlag. Pages 25-37.

2 Beaulieu, Julie, Gagné, Christian, and Parizeau, Marc. 2002. Lens system design and re-engineering experimentations with genetic algorithms and genetic programming. In Langdon, W. B., Cantu-Paz, E., Mathias, K., Roy, R., Davis, D., Poli, R., Balakrishnan, K., Honavar, V., Rudolph, G., Wegener, J., Bull, L., Potter, M. A., Schultz, A. C., Miller, J. F., Burke, E., and Jonoska, N. (editors). Proceedings of the 2002 Genetic and Evolutionary Computation Conference. San Francisco, CA: Morgan Kaufmann. Pages 155-162.

3 Comisky, William, Yu, Jessen, and Koza, John. 2000. Automatic synthesis of a wire antenna using genetic programming. Late Breaking Papers at the 2000 Genetic and Evolutionary Computation Conference, Las Vegas, Nevada. Pages 179-186.

4 Gardner, Leo R. 1998. Lens design problem summary: The solid glass lens. In Gardner, Leo R. and Thompson, Kevin P. (editors). International Optical Design Conference. Proceedings of SPIE. Volume 3482. Bellingham, WA: SPIE. Pages 2-8.

5 Gruau, Frederic. 1992. Cellular Encoding of Genetic Neural Networks. Technical report 92-21. Laboratoire de l'Informatique du Parallélisme. Ecole Normale Supérieure de Lyon. May 1992.

6 Jones, Lee W., Al-Sakran, Sameer H., and Koza, John R. 2005a. Automated design of a previously patented aspherical optical lens system by means of genetic programming. In Yu, Gwoing, Worzel, William, and Riolo, Rick (editors). Genetic Programming Theory and Practice III. New York: Springer. Chapter 3. Pages 33-48.

7 Jones, Lee W., Al-Sakran, Sameer H., and Koza, John R. 2005b. Automated synthesis of both the topology and numerical parameters for seven patented optical lens systems using genetic programming. In Mouroulis, Pantazis Z., Smith, Warren J., and Johnson, R. Barry (editors). Current Developments in Lens Design and Optical Engineering VI. Proceedings of SPIE. Volume 5874. Bellingham, WA: SPIE. Pages 24-38.

8 Juergens, Richard C. 2002. The 2002 IODC design problem - The diffractive simulator. In Manhart, Paul K. and Sasian, Jose M. (editors). International Optical Design Conference. Proceedings of SPIE. Volume 4832. Bellingham, WA: SPIE. Pages 473-485.
9 Koza, John R. 1993. Discovery of rewrite rules in Lindenmayer systems and state transition rules in cellular automata via genetic programming. Symposium on Pattern Formation (SPF-93), Claremont, California. February 13, 1993.

10 Koza, John R., Al-Sakran, Sameer H., and Jones, Lee W. 2005a. Automated re-invention of six patented optical lens systems using genetic programming. In Beyer, H.-G.; O'Reilly, U.-M.; Arnold, D.V.; Banzhaf, W.; Blum, C.; Bonabeau, E.W.; Cantu-Paz, E.; Dasgupta, D.; Deb, K.; Foster, J.A.; de Jong, E.D.; Lipson, H.; Llora, X.; Mancoridis, S.; Pelikan, M.; Raidl, G.R.; Soule, T.; Tyrrell, A.; Watson, J.-P.; Zitzler, E. (editors). Proceedings of the Genetic and Evolutionary Computation Conference GECCO2005. New York, NY: ACM Press. Pages 1953-1960.

11 Koza, John R., Al-Sakran, Sameer H., and Jones, Lee W. 2005b. Cross-domain features of runs of genetic programming used to evolve designs for analog circuits, optical lens systems, controllers, antennas, mechanical systems, and quantum computing circuits. In Lohn, Jason; Gwaltney, David; Hornby, Gregory; Zebulum, Ricardo; Keymeulen, Didier; and Stoica, Adrian (editors). Proceedings of the 2005 NASA/DoD Conference on Evolvable Hardware. Los Alamitos, CA: IEEE Computer Society Press. Pages 205-212.

12 Koza, John R., Bennett III, Forrest H, Andre, David, and Keane, Martin A. 1999. Genetic Programming III: Darwinian Invention and Problem Solving. San Francisco, CA: Morgan Kaufmann.

13 Koza, John R., Keane, Martin A., Streeter, Matthew J., Mydlowec, William, Yu, Jessen, and Lanza, Guido. 2003. Genetic Programming IV: Routine Human-Competitive Machine Intelligence. Kluwer Academic Publishers.

14 Prusinkiewicz, Przemyslaw, and Lindenmayer, Aristid. 1990. The Algorithmic Beauty of Plants. New York: SpringerVerlag.

15 Smith, Warren J. 1992. Modern Lens Design: A Resource Manual. Boston, MA: McGraw-Hill.

16 Smith, Warren J. 2000. Modern Optical Engineering. $3^{\text {rd }}$ edition. New York: McGraw-Hill.

17 Wilson, Stewart. W. 1987. The genetic algorithm and biological development. In Grefenstette, John J.(editor). Genetic Algorithms and Their Applications: Proceedings of the Second International Conference on Genetic Algorithms. Hillsdale, NJ: Lawrence Erlbaum Associates. Pages 247-251. 